\title{
Insertion of I-125 seed-loaded stent for inoperable hilar cholangiocarcinoma
}

\author{
Fu-Lei Gao ${ }^{1,2}$, Yong Wang ${ }^{3}$, Jian Lu³, Guang-Yu Zhu³, Xiang-Zhong Huang ${ }^{2}$, Dong-Qing Ren², Jin-He Guo ${ }^{1,3}$ \\ ${ }^{1}$ Nanjing Medical University, Nanjing, China \\ ${ }^{2}$ Department of Interventional Radiology, Affiliated Jiangyin Hospital of Medical College of Southeast University, Jiangyin, China \\ ${ }^{3}$ Centre of Interventional Radiology and Vascular Surgery, Department of Radiology, Zhongda Hospital, Medical School, \\ Southeast University, Nanjing, China
}

Videosurgery Miniinv 2021; 16 (4): 678-685

DOI: https://doi.org/10.5114/wiitm.2021.105530

\begin{abstract}
Introduction: Stent insertion is the most frequently used option to treat malignant biliary obstruction (MBO) patients. Hilar cholangiocarcinoma (HCCA) is the most common disease that causes hilar MBO.

Aim: To assess the clinical efficacy and long-term outcomes of I-125 seed-loaded stent (ISS) insertion for HCCA patients.

Material and methods: Consecutive patients with HCCA underwent either normal stent (NS) or ISS insertion between January 2017 and December 2019. The baseline and treatment data of these two groups were compared.

Results: During the period, a total of 93 patients with inoperable HCCA were divided into either NS $(n=48)$ or ISS $(n=45)$ insertion groups at our centre. Technical success rates of the NS and ISS insertion were $91.7 \%$ and $95.6 \%, r e-$ spectively $(p=0.733)$. Clinical success rates were $93.2 \%$ and $100 \%$ in the NS and ISS groups, respectively $(p=0.24)$. Stent dysfunction was observed in 11 and 8 patients in the NS and ISS groups, respectively $(p=0.47)$. The median stent patency was 143 days and 208 days in the NS and ISS groups, respectively $(p<0.001)$. All patients died in the follow-up period, with median survival duration of 178 days and 220 days in the NS and ISS groups, respectively $(p<0.001)$. ISS insertion was the only predictor of longer patency $(p=0.002)$ and survival $(p=0.01)$.

Conclusions: ISS insertion might achieve longer patency and overall survival in patients with inoperable HCCA as compared with NS insertion.
\end{abstract}

Key words: stent, I-125, hilar cholangiocarcinoma, patency, survival.

\section{Introduction}

Malignant biliary obstruction (MBO) usually arises in patients suffering from primary or metastatic hepatobiliary tumours [1-3]. Approximately $80 \%$ of MBO patients are not eligible for surgical resection, and as such palliative interventions are the only treatment option available for most patients [1-3]. Of the available palliative treatments, percutaneous or endoscopic stent insertion is the most frequently used to treat MBO patients [1-3].

Although stent insertion can achieve good short-term outcomes for patients with MBO, stent dysfunction remains a common outcome in these treated patients, limiting the long-term efficacy of this treatment strategy [4]. In order to overcome this shortcoming of normal stent (NS) insertion, many researchers have developed a novel biliary $1-125$ 
seed-loaded stent (ISS) [4]. The ISS not only effectively relieves the jaundice, but also can provide the brachytherapy to the tumour $[4,5]$. Previous metaanalyses also indicated that relative to NS insertion, ISS insertion was associated with longer survival and stent patency in inoperable MBO patients $[4,5]$. However, most previous studies included MBO patients with different cancer types and MBO sites, and therefore a risk of bias existed [6-9]. Hence, there is a clear need for a study comparing these two stent types in patients with a single type of cancer.

\section{Aim}

Hilar MBO is an important part of MBO [10]. Hilar cholangiocarcinoma (HCCA) is the most common disease that causes hilar MBO [11-14]. Herein, we assessed the clinical and long-term efficacy of ISS insertion for HCCA patients.

\section{Material and methods}

Our Institutional Review Board approved the present retrospective study. Because the study was retrospective, the requirements for written informed consent of the patients were waived.

\section{Study design}

Consecutive patients with HCCA underwent either ISS or NS insertion between January 2017 and December 2019. The patients who were treated before December 2018 underwent NS insertion, while those who were treated thereafter underwent ISS insertion.

Study inclusion criteria were as follows: (a) patients with a diagnosed HCCA, (b) inoperable cases, (c) patients showing evidence of obstructive jaundice, and (d) Eastern Cooperative Oncology Group performance status (ECOG PS) < 4. Excluded patients met the following criteria: (a) patients who underwent post-operative external radiotherapy, (b) patients in whom prior biliary stent insertion or drainage were performed, and (c) patients suffering from serious dysfunction of the pulmonary, renal, cardiac, or coagulatory systems. Following stenting, chemotherapy was permitted for all patients.

\section{Diagnosis}

HCCA was diagnosed based on patients' clinical symptoms and the results of their abdominal com- puted tomography (CT) and magnetic resonance imaging (MRI) analyses. Pathological HCCA diagnosis was confirmed via biopsy.

\section{NS insertion}

All procedures were performed with fluoroscopic guidance. A 21G Chiba needle (Cook, IN, USA) was used to puncture the right intrahepatic biliary tract using combined ultrasonic and fluoroscopic guidance, with cholangiography being conducted to image the obstruction. A 4F VER catheter (Cordis, Warren, FL, USA) with a 0.035-inch normal guide wire (Terumo, Tokyo, Japan) was then employed to pass through the obstruction. When the catheter and guide wire entered the duodenum, a 0.035-inch stiff guide wire (Cook) was used to replace the normal guide wire. The stent (Micro-Tech, Nanjing, China) was released at the site of the obstruction via the stiff guide wire.

\section{ISS insertion}

An ISS consisted of two overlapped parts: an inner normal bare stent (Micro-Tech) and an outer seeds-loaded stent. Each I-125 seed (Chinese Atomic Energy Science Institution, Beijing, China) was attached to the stent surface before treatment. Individual I-125 seeds (dimensions: $4.5 \mathrm{~mm}$ long, $0.8 \mathrm{~mm}$ diameter) emitted low-energy $35.5-\mathrm{keV}$ $\gamma$-rays with a 59.6-day half-life.

A 21G Chiba needle (Cook) was utilized to puncture the right intrahepatic biliary tract. Following the coaxial dilation with a balloon dilator catheter, the outer seed-loaded stent was inserted into a $10 \mathrm{~F}$ sheath and was then introduced and deployed into the obstructed site over a stiff guide wire. After that, the inner stent was instantly placed to overlap the outer seed-loaded stent (Photo 1).

\section{Post-operative treatment}

Postoperatively, a temporary biliary catheter (Cook) was inserted into each patient and drained for 3 days; all patients were administered preventive anti-inflammatory medications and haemostasis for 5 days.

\section{Assessment}

Technical success for stenting was confirmed based on the extent of obstruction elimination, which 

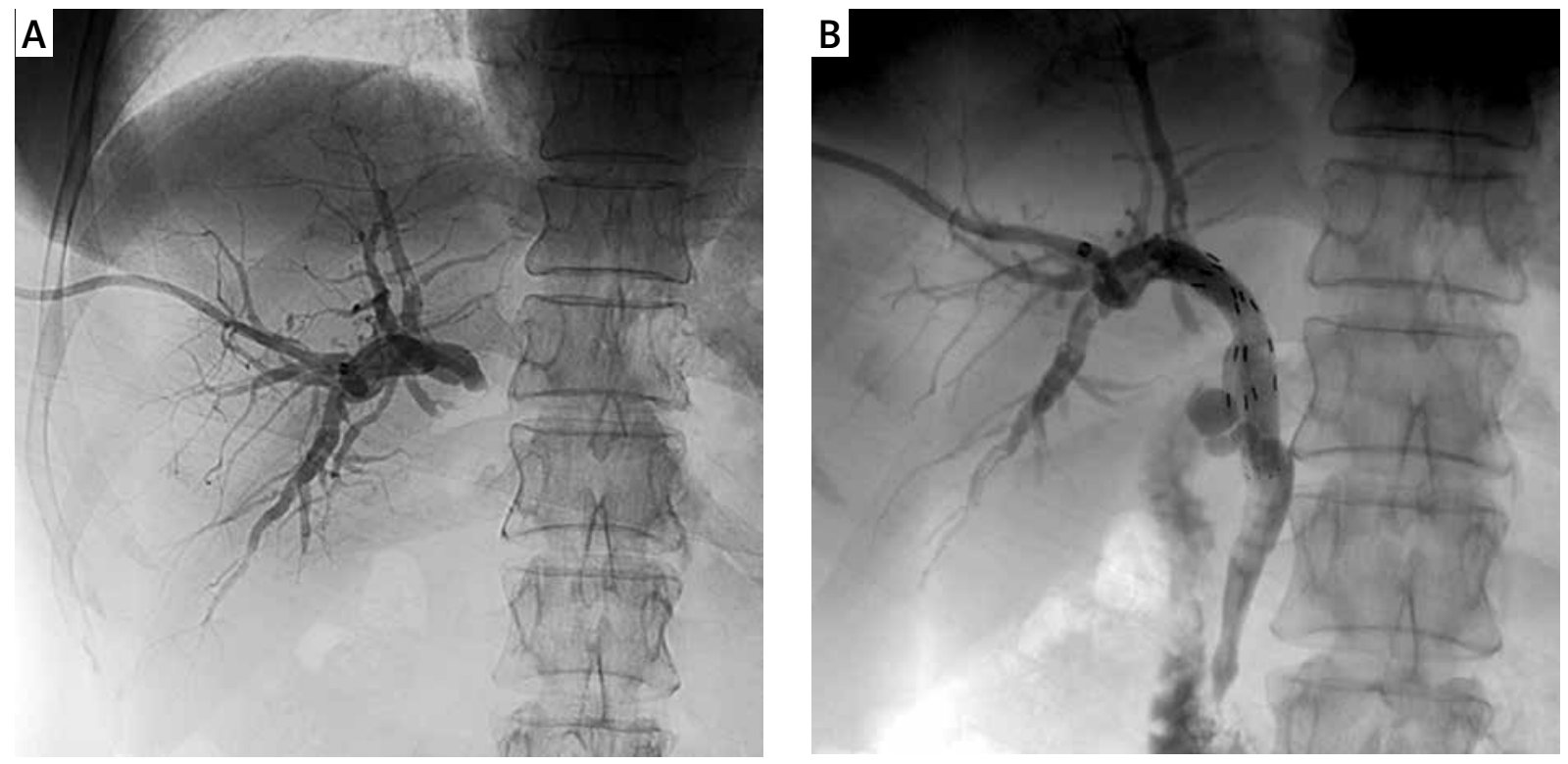

Photo 1. ISS insertion for a patient with HCCA. A - The pre-stenting cholangiography showed the obstruction located at the hilar site. $\mathbf{B}$ - The stent was placed in the right intra-hepatic and common biliary tracts

enabled smooth contrast medium flow through the stent [10-12]. Clinical success was deemed to have occurred when total bilirubin (TBIL) levels fell to $<70 \%$ of the preoperative baseline within a 2-week period following stent insertion [3]. Stent dysfunction was confirmed based on recurrent cases of cholangitis and/or jaundice as a result of either re-obstruction or migration [10-12]. Stent patency was the duration from stent insertion to patient death or stent dysfunction. Overall survival was the time period from stent insertion until patient death.

All patients underwent postoperative physical examination, $\mathrm{CT}$ examination, and liver function tests after 2 weeks, 1, 3, and 6 months, and every 6 months thereafter.

\section{Statistical analysis}

SPSS v16.0 (SPSS, Inc., IL, USA) was employed for all statistical testing. Continuous and categorical variables were respectively analysed via $t$-tests and $\chi^{2}$ tests. Patient survival and stent patency were compared using the Kaplan-Meier curve and logrank tests. A multivariate Cox regression analysis was used for identification of the factors related to patient survival. All variables that achieved a $p$-value of $<0.1$ in the initial univariate analysis were incorporated into the subsequent multivariate model. $P<0.05$ was maintained as the statistical significance threshold.

\section{Results}

\section{Patients}

During this study period, 93 patients with inoperable HCCA were divided to either NS $(n=48)$ or ISS ( $n=$ 45) insertion groups at our centre. No patient was lost to follow-up. Six and 7 patients underwent trans-hepatic arterial chemotherapy infusion after stent insertion in the NS and ISS groups, respectively $(p=0.643)$.

\section{Technical success}

Technical success rates for the NS and ISS insertion groups were $91.7 \%$ and $95.6 \%$, respectively $(p=0.733)$. The baseline data for patients who successfully received the stent insertion are shown in Table I. Neither group exhibited procedure-related complications. Each patient received unilateral stent insertion with 1 stent. Stent diameter and length were $8 \mathrm{~mm}$ and $50-70 \mathrm{~mm}$, respectively. The technical failure of the stent insertion was found in $6 \mathrm{pa}$ tients because the guide wire was unable to pass the obstructed site. We performed a second attempt after failure of initial stenting. However, the guide wire still could not pass the obstructed site. These 6 patients were given an extra-biliary drainage catheter.

\section{Clinical success}

Clinical success rates were $93.2 \%$ and $100 \%$ in the NS and ISS groups, respectively $(p=0.24$, 
Table I. Patient characteristics in the two groups

\begin{tabular}{|c|c|c|c|}
\hline Variable & Normal stent & I-125 seed-loaded stent & $P$-value \\
\hline Patients number & 44 & 43 & - \\
\hline Age [years] & $66.2 \pm 12.3$ & $66.9 \pm 10.1$ & 0.772 \\
\hline Male/female & $18 / 26$ & $16 / 27$ & 0.724 \\
\hline ECOG PS (II/III) & $13 / 31$ & $18 / 25$ & 0.236 \\
\hline Tumour stage (II/III/IV) & 19/18/7 & $19 / 16 / 8$ & 0.917 \\
\hline Bismuth type (I/II/III/IV) & $6 / 13 / 18 / 7$ & $7 / 16 / 15 / 5$ & 0.806 \\
\hline \multicolumn{4}{|l|}{ TBIL [ $\mu \mathrm{mol} / \mathrm{l}]:$} \\
\hline Before & $208.0 \pm 103.9$ & $226.2 \pm 120.9$ & 0.454 \\
\hline After & $101.0 \pm 63.4$ & $101.4 \pm 57.9$ & 0.974 \\
\hline$P$-value & $<0.001$ & $<0.001$ & - \\
\hline \multicolumn{4}{|l|}{ AST [U/I]: } \\
\hline Before & $147.6 \pm 97.0$ & $160.2 \pm 130.7$ & 0.611 \\
\hline After & $69.4 \pm 42.8$ & $58.8 \pm 32.0$ & 0.197 \\
\hline$P$-value & $<0.001$ & $<0.001$ & - \\
\hline \multicolumn{4}{|l|}{ ALT [U/l]: } \\
\hline Before & $132.6 \pm 90.0$ & $165.2 \pm 127.6$ & 0.171 \\
\hline After & $64.4 \pm 41.6$ & $61.5 \pm 39.6$ & 0.579 \\
\hline$P$-value & $<0.001$ & $<0.001$ & - \\
\hline Albumin [g/l] & $34.5 \pm 3.7$ & $35.0 \pm 3.8$ & 0.736 \\
\hline Carbohydrate antigen-199 [U/I] & $405.2 \pm 338.8$ & $448.5 \pm 414.8$ & 0.579 \\
\hline
\end{tabular}

ECOG PS - Eastern Cooperative Oncology Group performance status, TBIL - total bilirubin, AST - aspartate transaminase, ALT - alanine aminotransferase.

Table II). The improvements of total bilirubin (TBIL), aspartate transaminase (AST), and alanine aminotransferase (ALT) levels in both groups are shown in Table I.

\section{Stent patency}

Stent dysfunction was observed in 11 and 8 patients in the NS and ISS groups, respectively $(p=0.47$,
Table II). Tumour growth was the cause of all stent dysfunction. In the NS group, 10 patients had a second stent inserted and 1 patient underwent in-stent biliary drainage catheter insertion. In the ISS group, 6 patients had a second stent inserted and 2 patients underwent in-stent biliary drainage catheter insertion. The median stent patency was 143 days and 208 days in the NS and ISS groups, respectively $(p<$ 0.001 , Figure 1). The 3-, 6-, and 12-month patency

Table II. Comparison of outcomes between the two groups

\begin{tabular}{|lccc|}
\hline Variable & Normal stent & I-125 seeds-loaded stent & $P$-value \\
\hline Technical success & $44 / 48(91.7 \%)$ & $43 / 45(95.6 \%)$ & 0.733 \\
\hline Clinical success & $41 / 44(93.2 \%)$ & $43 / 43(100 \%)$ & 0.240 \\
\hline Stent dysfunction & $11 / 44(25.0 \%)$ & $8 / 43(18.6 \%)$ & 0.470 \\
\hline Complications: & & & 0.959 \\
\hline Cholangitis & $8 / 44(18.1 \%)$ & $8 / 43(18.6 \%)$ & 0.628 \\
\hline Cholecystitis & $3 / 44(6.8 \%)$ & $1 / 43(2.3 \%)$ & 0.643 \\
\hline Post-operative chemotherapy & 6 & 7 & $<0.001$ \\
\hline Patency [days] & 143 & 208 & $<0.001$ \\
\hline Overall survival [days] & 178 & 220 & \\
\hline
\end{tabular}




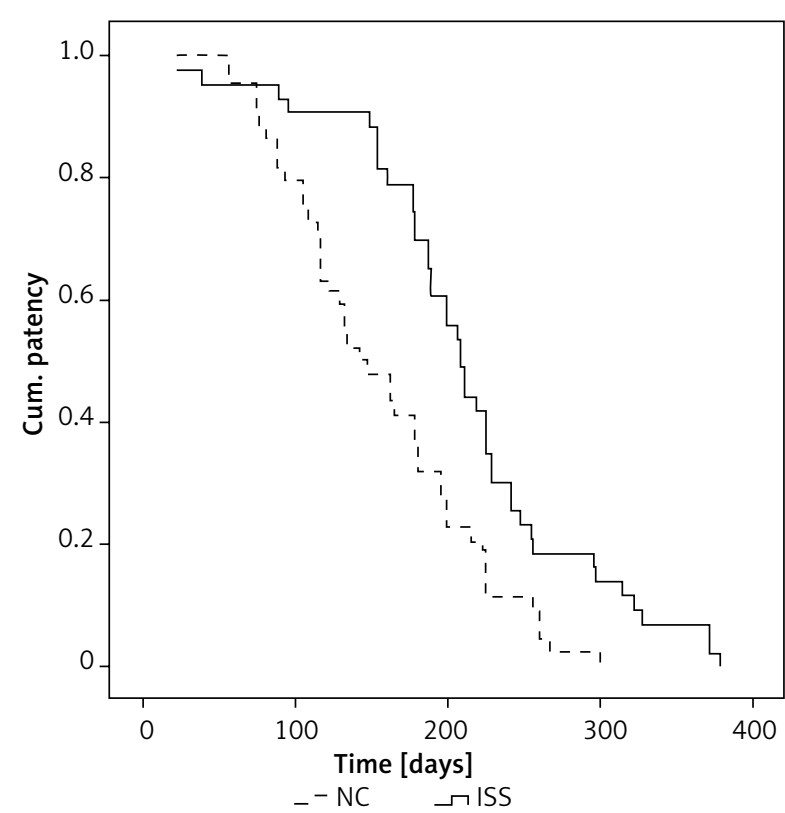

Figure 1. Kaplan-Meier curve demonstrated significantly longer patency after ISS insertion

rates were $81.8 \%$ and $93.0 \%, 36.4 \%$ and $69.8 \%$, and $0 \%$ and $7 \%$ in the NS and ISS groups, respectively.

A univariate Cox-regression analysis revealed that male sex $(H R=1.555 ; 95 \%$ confidential interval: $0.990-2.442 ; p=0.055)$, lower carbohydrate antigen-199 level $(\mathrm{HR}=1.001 ; 95 \% \mathrm{Cl}: 1.000-1.001$; $p=0.057)$, and the use of ISS ( $\mathrm{HR}=0.464 ; 95 \% \mathrm{Cl}$ : $0.297-0.724 ; p=0.001)$ were associated with longer patency. When these 3 factors were included in multivariate analysis, it was found that use of ISS was the only predictor of longer patency $(\mathrm{HR}=0.483 ; 95 \% \mathrm{Cl}$ : 0.307-0.795; $p=0.002$, Table III). The post-operative trans-hepatic arterial chemotherapy infusion was not associated with longer patency $(p=0.520)$.

\section{Survival}

All patients died in the follow-up period due to tumour progression, with median survival du-

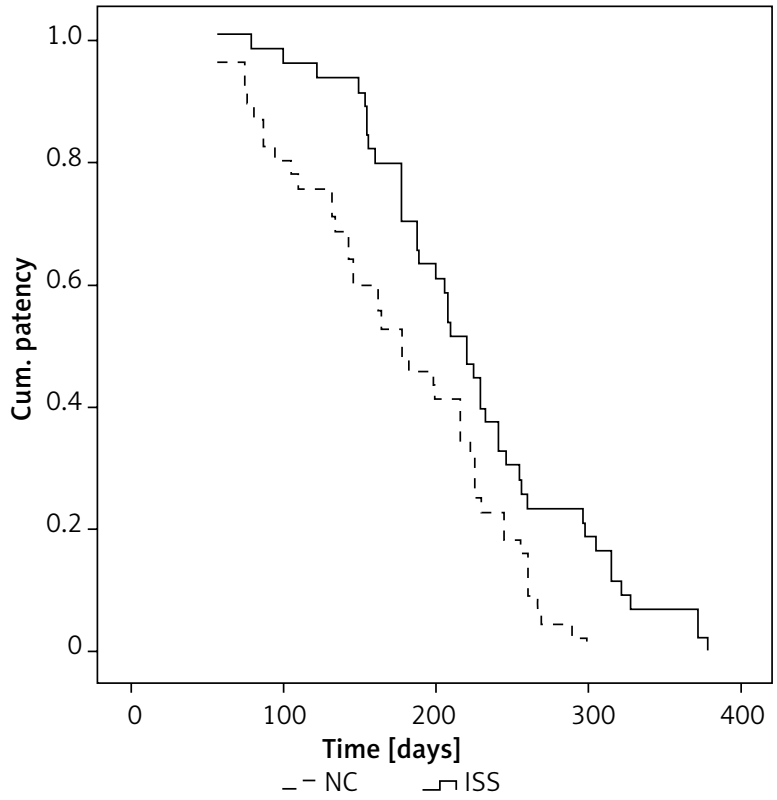

Figure 2. Kaplan-Meier curve demonstrated significantly survival after ISS insertion

ration of 178 days and 220 days in the NS and ISS groups, respectively ( $p<0.001$, Figure 2 ). The 3-, 6-, and 12 -month survival rates were $81.8 \%$ and $97.7 \%$, $47.7 \%$ and $69.8 \%$, and $0 \%$ and $7 \%$ in the NS and ISS groups, respectively.

A univariate Cox regression analysis indicated that male sex $(\mathrm{HR}=1.576 ; 95 \% \mathrm{Cl}: 0.997-2.492$; $p=0.052$ ), lower carbohydrate antigen-199 level $(\mathrm{HR}=1.001 ; 95 \% \mathrm{Cl}: 1.000-1.001 ; p=0.049)$, and the use of ISS ( $\mathrm{HR}=0.525 ; 95 \% \mathrm{Cl}: 0.334-0.825$; $p=0.005)$ were associated with longer survival. When these 3 factors were included in multivariate analysis, it was found that use of ISS was the only predictor of longer survival $(\mathrm{HR}=0.542$; 95\% Cl: 0.341-0.862; $p=0.01$, Table IV). The post-operative trans-hepatic arterial chemotherapy infusion was not associated with longer survival $(p=0.949)$.

Table III. Predictors of stent patency after stent insertion

\begin{tabular}{|lcccccc|}
\hline Variable & \multicolumn{3}{c}{ Univariate analysis } & \multicolumn{3}{c|}{ Multivariate analysis } \\
\cline { 2 - 7 } & Hazard ratio & $95 \% \mathrm{Cl}$ & $P$-value & Hazard ratio & $95 \% \mathrm{Cl}$ & $P$-value \\
\hline Gender & 1.555 & $0.990,2.442$ & 0.055 & 1.263 & $0.792,2.013$ & 0.326 \\
\hline CA-199 & 1.001 & $1.000,1.001$ & 0.057 & 1.000 & $1.000,1.001$ & 0.092 \\
\hline Use of ISS & 0.464 & $0.297,0.724$ & 0.001 & 0.483 & $0.307,0.795$ & 0.002 \\
\hline
\end{tabular}

ISS-I-125 seed-loaded stent, CA-199 - carbohydrate antigen-199. 
Table IV. Predictors of survival after stent insertion

\begin{tabular}{|lcccccc|}
\hline Variable & \multicolumn{3}{c}{ Univariate analysis } & \multicolumn{3}{c|}{ Multivariate analysis } \\
\cline { 2 - 8 } & Hazard ratio & $95 \% \mathrm{Cl}$ & $P$-value & Hazard ratio & $95 \% \mathrm{Cl}$ & $P$-value \\
\hline Gender & 1.576 & $0.997,2.492$ & 0.052 & 1.242 & $0.762,2.023$ & 0.385 \\
\hline CA-199 & 1.001 & $1.000,1.001$ & 0.049 & 1.000 & $1.000,1.001$ & 0.093 \\
\hline Use of ISS & 0.525 & $0.334,0.825$ & 0.005 & 0.542 & $0.341,0.862$ & 0.01 \\
\hline
\end{tabular}

ISS - I-125 seed-loaded stent, CA-199-carbohydrate antigen-199.

\section{Complications}

A total of 8 and 8 patients in the NS and ISS groups, respectively, suffered from cholangitis ( $p=$ 0.959 , Table II). The cholangitis occurred after stent dysfunction, and they were resolved by re-intervention of the stent dysfunction. Three and 1 patient/s in the NS and ISS groups, respectively, suffered from cholecystitis ( $p=0.628$, Table II). Cholecystitis was managed by conservative treatment (analgesia, spasmolysis, and anti-inflammatory).

\section{Discussion}

Herein, we compared the clinical and long-term efficacy of NS or ISS insertion in HCCA patients. High and comparable technical $(91.7 \%$ vs. $95.6 \%$, $p=0.733)$ and clinical ( $93.2 \%$ vs. $100 \%, p=0.24)$ success rates were observed in both the groups, which suggests that both NS and ISS are reliable and safe for providing instant palliative relief to patients with inoperable HCCA.

Some researchers have reported their clinical experience of laparoscopic radical resection of HCCA with the 3-year overall survival and disease-free survival rates of $49.1 \%$ and $47.0 \%$, respectively [10]. Although these results were optimal, 29-40\% HCCA patients had locally advanced or metastatic diseases at diagnosis, making them ineligible for surgical resection [10]. Moreover, at the time of the curative-intent operation, only $50-60 \%$ of patients were ultimately found to be resectable. Therefore, most of the HCCA patients are suitable for palliative stent insertion [1-3].

Although some publications have shown the clinical effectiveness of ISS insertion for inoperable MBO, these studies included patients with multiple cancer types and/or obstructed sites [4-9]. The multiple cancer types and/or obstructed sites caused high risk of selection bias. Compared to the previous studies, our study included a unique obstructed site (hilar MBO) with a single cancer type (HCCA). Al- though our study is a retrospective study, the single cancer type might reduce the risk of selection bias.

Two types of ISSs have been reported previously [4-9]. As well as the type that we used in this study, there is another type of radioactive stent which is made by a NS with a radioactive seed strand [7-9]. The radioactive seed strand is made by a $4 \mathrm{~F}$ catheter combined with multiple I- 125 seeds [7-9]. When placing the stent, the radioactive seed strand is placed beside or within the stent [7-9]. Compared to the ISS in the present study, the radioactive seed strand has a simple manufacturing process. However, the primary biliary adenocarcinoma usually grows surround the biliary wall; therefore, the radioactive seed strand may not give a comprehensive treatment of HCCA.

Unlike some previous results suggesting bilateral stent insertion for patients with HCCA [11-14], this study used unilateral stent insertion for HCCA. Although some meta-analyses have demonstrated that bilateral stenting can provide a longer stent patency than unilateral stenting, those meta-analyses both included percutaneous and endoscopic biliary stenting $[13,14]$. The clinical effectiveness between percutaneous and endoscopic biliary stenting for MBO was different [1]. This study used a percutaneous approach to place the stents. Some recent studies demonstrated that percutaneous unilateral and bilateral metal stenting are similarly effective for the treatment of patients with hilar MBO $[3,15]$. Therefore, we believe that percutaneous unilateral stenting is sufficient to treat patients with HCCA.

Furthermore, the most effective method of prolonging stent patency is to prevent the tumour growth [16]. Previously, conventional chemotherapy and radiotherapy were always used to prevent tumour growth after stent insertion [16, 17]. As compared to conventional chemotherapy and radiotherapy, ISSs offer significant advantages, such as the ease of manipulation and provision of directly contacting the surface of the tumour, permitting 
prolonged, sustained administration of low doses of radiation [4-8].

In this present study, the stent patency was significantly longer in the ISS group relative to that in the NS group (208 days vs. 143 days; $p<0.001$ ), with similar overall stent dysfunction rates $(p=0.47)$. Definitely, ISSs could not completely prevent the tumour growth because not all patients were sensitive to intra-luminal brachytherapy all the time. However, ISSs could prolong the time-to-stent dysfunction. The Cox regression analysis also found that ISS insertion was an independent predictor of longer stent patency.

We found that the ISS group patients survived significantly longer than the NS group patients (220 days vs. 178 days; $p<0.001$ ). This advantage was in accordance with the previous studies of ISS insertion for patients with MBO [6-9]. I-125 seeds can continuously release $X$ and $\gamma$ rays to effectively kill tumour cells and inhibit tumourigenesis [18]. Furthermore, the percentages of CD3+ T, CD4+ T, natural killer, and regulatory $T$ cells significantly increased in the peripheral blood of tumour patients after I-125 seed placement [18]. The median 220-day survival duration in the ISS group was consistent with that reported in previous studies (202-355 days) on MBO patients who underwent ISS insertion [6-8].

Based on the Cox regression analysis, the post-operative trans-hepatic arterial chemotherapy infusion was not associated with longer patency $(p=0.520)$ or survival $(p=0.949)$. These results might be attributed to the limited sample size of the patients who underwent post-operative trans-hepatic arterial chemotherapy. Furthermore, Bismuth type and tumour stage were not found to be associated with survival. The included patients were all inoperable cases; therefore, there might be no significant difference in patients' body condition between different Bismuth types and tumour stages.

No significant differences in complications were observed between these groups, which indicates that the ISSs did not aggravate the complication rate. Only $18.4 \%(16 / 87)$ of patients in the present study experienced cholangitis. This low rate was probably due to the fact that all patients had a temporary drainage catheter inserted for up to 5 days post-stenting.

We identified multiple limitations in this study. First, this analysis was retrospective and hence susceptible to selective bias. However, the comparable baseline data and unique cancer type might reduce the risk of bias. Second, we could not measure the ISS dosimetry accurately owing to the lack of data on dedicated measurements for luminal organs. Third, the sample size of this study was not large, and no sample size-related power calculations could be performed owing to the retrospective nature of this study. Furthermore, we did not stratify the data of stent patency and overall survival with tumour stage due to the limited sample size. Considering these limitations, it is important to validate our findings in future prospective studies.

\section{Conclusions}

ISS insertion might achieve longer patency and overall survival in patients with inoperable HCCA as compared with NS insertion.

\section{Acknowledgments}

This study was supported by National Natural Science Foundation of China (81971716).

\section{Conflict of interest}

The authors declare no conflict of interest.

\section{References}

1. Zhao XQ, Dong JH, Jiang K, et al. Comparison of percutaneous transhepatic biliary drainage and endoscopic biliary drainage in the management of malignant biliary tract obstruction: a meta-analysis. Dig Endosc 2015; 27: 137-45.

2. Almadi MA, Barkun JS, Barkun AN. Stenting in malignant biliary obstruction. Gastrointest Endosc Clin N Am 2015; 25: 691-711.

3. Fu YF, Zhou WJ, Shi YB, et al. Percutaneous stenting for malignant hilar biliary obstruction: a randomized controlled trial of unilateral versus bilateral stenting. Abdom Radiol 2019; 44: 2900-8.

4. Sha KH, Liu TG, Yang F, et al. Irradiation stent insertion for inoperable malignant biliary obstruction: a meta-analysis of randomized controlled trials. Abdom Radiol 2020; doi: 10.1007/ s00261-020-02851-6.

5. Xu X, Li J, Wu J, et al. A systematic review and meta-analysis of intraluminal brachytherapy versus stent alone in the treatment of malignant obstructive jaundice. Cardiovasc Intervent Radiol 2018; 41: 206-17.

6. Zhu HD, Guo JH, Huang M, et al. Irradiation stents vs. conventional metal stents for unresectable malignant biliary obstruction: a multicenter trial. J Hepatol 2018; 68: 970-7.

7. Jiao D, Wu G, Ren J, et al. Study of self-expandable metallic stent placement intraluminal 125 I seed strands brachytherapy of malignant biliary obstruction. Surg Endosc 2017; 31: 49965005. 
8. Chen $G$, Zhang M, Sheng YG, et al. Stent with radioactive seeds strand insertion for malignant hilar biliary obstruction. Minim Invasive Ther Allied Technol 2020; doi: 10.1080/ 13645706.2020 .1735446$.

9. Li S, Li B, Li L, et al. The efficacy of the combination of percutaneous transhepatic biliary drainage and $125 \mathrm{I}$ stranded seeds for malignant bile duct obstruction treatment. J Contemp Brachytherapy 2020; 12: 225-32.

10. Li J, Xiong Y, Yang G, et al. Complete laparoscopic radical resection of hilar cholangiocarcinoma: technical aspects and longterm results from a single center. Videosurgery Miniinv 2021; 16: 62-75.

11. Law R, Baron TH. Bilateral metal stents for hilar biliary obstruction using a 6 Fr delivery system: outcomes following bilateral and side-by-side stent deployment. Dig Dis Sci 2013; 58: 2667-72.

12. Naitoh I, Hayashi K, Nakazawa T, et al. Side-by-side versus stentin-stent deployment in bilateral endoscopic metal stenting for malignant hilar biliary obstruction. Dig Dis Sci 2012; 57: 3279-85.

13. Li M, Wu W, Yin Z, et al. Unilateral versus bilateral biliary drainage for malignant hilar obstruction: a systematic review and meta-analysis. Zhonghua Gan Zang Bing Za Zhi 2015; 23: 118-23.

14. Ashat M, Arora S, Klair JS, et al. Bilateral vs unilateral placement of metal stents for inoperable high grade hilar biliary strictures: a systemic review and meta-analysis. World J Gastroenterol 2019; 25: 5210-9.

15. Fu YF, Xu YS, Shi YB, et al. Percutaneous metal stenting for malignant hilar biliary obstruction: a systematic review and meta-analysis of unilateral versus bilateral stenting. Abdom Radiol 2021; 46: 749-56.

16. Li TF, Chen C, Han XW, et al. Clinical efficacy of metallic biliary stents combined with different anti-cancer treatments in the management of bile duct cancer. Hepatogastroenterology 2014; 61: 22-6.

17. Boulay BR, Birg A. Malignant biliary obstruction: from palliation to treatment. World J Gastrointest Oncol 2016; 8: 498-508.

18. Pang Q, Zhou L, Hu XS, et al. Biliary stenting alone versus biliary stenting combined with 1251 particles intracavitary irradiation for the treatment of advanced cholangiocarcinoma. Sci Rep 2019; 9: 11348 .

Received: 4.01.2021, accepted: 5.02.2021. 